\title{
Considerations regarding food supplements and plant extracts used in preventing and combating SARS-CoV-2
}

\author{
Daniela Cîrţină ${ }^{1}$, Larisa Marina Elisabeth Chirigiu ${ }^{1}$ \\ 1"Constantin Brâncusi" University of Tg-Jiu, Faculty of Medical and Behavioral \\ Sciences, No. 4, Tineretului St., Tg-Jiu, Romania \\ E-mail: danielacirtina @ gmail.com
}

\begin{abstract}
COVID-19, the disease caused by the SARS-CoV-2 virus, is an absolute novelty for the medical systems around the world, both in the variety of forms of manifestation, the effects on the human body and in the economic and social consequences produced globally. This paper presents some scientific remedies useful in SARS-CoV-2 infection, scientifically confirmed. Their maximum effect on the body can be achieved, only by the combination with antiviral drugs, obtaining in the end the treatment of COVID-19.
\end{abstract}

Keywords: food supplements, plants extracts, SARS-CoV-2

\section{Introduction}

In the COVID-19 disease, they have been used alongside the antiviral drugs, allopathic, antiinflammatory, nebulizing, antithermal, antithrombotic, anticoagulant, antibiotic, hypoglycemic, antiacid, antiemetic, antihypertensive, anxiolytic, immune antimodulatory, supplemental and herbal medications both in the incubation phase as well as in the symptomatic one (with an inflamed organism and covered by a form called cytokine storm). Since the outbreak of the pandemic in 2020 , the emphasis has been on the benefits of vitamin supplements to prevent and treat COVID-19, as it is known that dietary supplements can help maintain a healthy immune system. [1]

It has been observed that this disease is somehow slowed down in any phase of it, even in the asymptomatic phase by certain plant extracts, natural nutritional supplements that have no side effects, in certain doses. Specialist studies conducted in countries such as the United Kingdom, USA and Sweden have indicated that the consumption of probiotics, Omega-3, multivitamins or vitamin $\mathrm{D}$ has been associated with a lower risk of infection with SARS-CoV-2. Sometimes, these substances are used as real medicines, although they are just foods, concentrated supplements, but used for a long time, they even gave the desired effect. These include: vitamin D3, vitamin C, selenium, zinc, omega 3 and melatonin. Among the plants, the following are used: dandelion, boswellia, ginseng, valerian, licorice, passiflora, rhodiola, ashwaganda, lemon balm, hawthorn flowers, turmeric, echinacea, wormwood, green tea extract, olive leaf extract and hemoderivatives of black poplar, hornbeam and blackcurrant. [2]

In COVID-19 disease, it has been observed that the reservoir of viral replication in an infected body is the mucous membranes: nasal, buccal and pharyngeal. Then the virus enters the upper respiratory tract to the lungs. A number of clinical trials in China claim that the administration of traditional herbal medicines has led to the recovery of $90 \%$ of patients treated. At the same time, some traditional herbal medicines have prevented SARS-CoV-2 infection in healthy people and improved the health of patients with mild or severe symptoms. [3] 
2.Food supplements that increase immunity and reduce the chances of infection with the new coronavirus. Vitamins

More and more specialized studies show that vitamin D has an effect on the immune system and stops the spread of infections, being used in the treatment plan of COVID-19. Studies in Spain on COVID-19 infection conclude that $80 \%$ of coronavirus patients have vitamin D deficiencies.

Vitamin D is a hormone produced by the kidneys that controls the concentration of calcium in the blood and adjusts the immune system. This could help in the fight with COVID-19, to prevent certain strong reactions, the so-called cytokine storm, which occurs in most severe cases of coronavirus. Vitamin D deficiencies increase the risks for the development of autoimmune diseases, such as asthma, HIV, various types of cancer. At the same time, vitamin D supplementation can reduce the risk of respiratory infections. [4,5]

Along with the skin and liver, the following products are also rich in vitamin D, respectively multivitamins D: egg yolk, fish oil, brewer's yeast, mushrooms, cereals, orange juice.

The structure of vitamin D3

All vitamins $\mathrm{D}$ come from the corresponding sterols by opening their $\beta$ cycle and forming a system of conjugated double bonds. Thus, vitamin D3 is formed from 7-dehydrocholesterol, which has the following chemical structure:

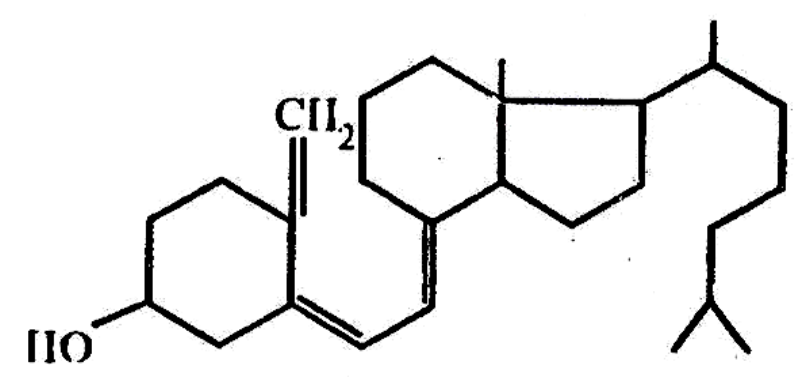

Cholecalciferol (Vitamin D3)

The active form of cholecalciferol (vitamin D3) is actually 1,25-dihydrocholecalceferol resulting from the hydroxylation of chalceferol in liver cell microsomes at position 25 , then it is hydroxylated at position 1, the process taking place in the mitochondria of renal cells and resulting in 1,25dihydroxycholec, which behaves like a hormone. [6]

Use of vitamin $D 3$ in the control of COVD-19

COVD-19 patients, with severe forms, have been found to have a small amount of D3 in the body. In the intensive care unit, it was observed that at the intubated patients, who were given 100,000 U.I. for at least 5 days, the number of intubation days decreased greatly, and the hemoglobin level increased as well. For the absorption of vitamin D3, it was recommended to infuse a large amount of serum + magnesium. This vitamin is fat-soluble, is administered after a meal, the duration of oral administration is 14 days, with high doses in the first days of the disease, and then decreases $(5,000$ IU / day), using minimum doses even three months.

\section{Vitamin $C$ and the immunity}

The $\mathrm{C}$ vitamin has anti-inflammatory properties, influences cellular immunity and vascular integrity, which is why it helps in situations where the body would be subjected to stress, as in the case of infection with the new coronavirus. Vitamin C helps the body to function properly and contributes to the formation of collagen, a protein that helps heal wounds, keeps skin and bones healthy. Vitamin $\mathrm{C}$ also increases the level of iron absorption in the body.

All green vegetables and fruits contain vitamin $C$. Among the vegetables rich in vitamin $C$ we can find: peppers, Brussels sprouts, broccoli, cucumbers and tomatoes. Fruits rich in vitamin $\mathrm{C}$ are: oranges, lemons, strawberries, currants, raspberries, blackberries, kiwis and strawberries. [7] 
The physiological role of the ascorbic acid

The ascorbic acid is $\gamma$-lactone of the 2,3-endiol-L-gluconic acid and has strong redox properties in solution. By oxidation it passes into dehydroascorbic acid, which participates easily in the reduction reaction by reversibly passing into ascorbic acid. The ascorbic acid has an asymmetric carbon atom (C5). Only the L isomer and the reduced form shows biological activity. The acidic character is given by endiolic groups that can dissociate with the release of protons. Vitamin $\mathrm{C}$ has a highly reactive furanosic structure; by substitutions in the furanosic cycle, the biological activity decreases and even disappears. The ascorbic acid can be biosynthesized from glucose by the animal body except humans, primates and guinea pigs. These mammals do not have the enzyme Lgluconoxidase in the liver, which is involved in the biosynthesis of vitamin C.

The dehydroascorbic acid participates in various metabolic processes due to its ability to form a reversible redox system. The ascorbic acid stimulates the metabolism of carbohydrates, lipids, glycoproteins and many amino acids; it behaves in some enzymatic systems as a hydrogen transporter intervening in reactions catalyzed by oxidoreductases (dehydrogenases, catalases, peroxidases, cytochromoxidases etc.). In the cellular respiration, the ascorbic acid reacts with oxygen reducing it to water or hydrogen peroxide. It intervenes in the normal metabolism of some amino acids (such as tyrosine, phenylalanine), in hydroxylation reactions of some amino acids, aromatic compounds and fatty acids and in the formation in the body of ketone compounds such as 17-ketosteroid hormones or of the $\alpha$-ketoglutaric acid from the citric acid. Vitamin C stimulates the activity of deaminases and other enzymes of liver and muscle glycogenase. It has a role in the biosynthesis of mucopolysaccharides, in the formation of collagen fibers, intervening in the conversion of hydroxyproline necessary for the biosynthesis of the collagen molecule, it intervenes in the formation of bone tissue. Vitamin C has antitoxic action, increasing the body's resistance to infections, promotes blood clotting, transport and iron storage, participating in the proper functioning of the cardiovascular system and nervous system. [8]

Use of vitamin $C$ in the control of COVD-19

Vitamin C has been used successfully especially in the intensive care and COVID rooms, in therapy in high doses continuously, intravenously $20 \mathrm{~g} /$ day and for the unintubated patients, in low doses (causes diarrhea), having a good antiviral, anti-inflammatory action; it is known that the immune cells and kidney cells consume the highest quantity of vitamin $\mathrm{C}$ in the body, the excess leading to the formation of kidney stones. It helps the immune system by the antioxidant effect on the tissues and by inhibiting the inflammatory effect of polysaccharides involved in the so-called cytokine storm in very serious conditions of SARS-Cov-2 infection, which ultimately results in the destruction of the lungs. [9]

\section{The role of the trace elements in increasing the immunity \\ Selenium}

It helps the human body because it is an essential trace element from selenium proteins with a protective role, meaning that it inhibits SARS-CoV-2 proteases.

\section{Zinc}

This element is generally a good stimulant of the deficient immune system in certain diseases, such as diabetes, obesity, cardiovascular disease. In COVID-19, it is beneficial, reducing the replication of the virus (50 $\mathrm{mg} /$ day) as well as pneumonia. In colds, zinc reduces the duration of the disease, by being present in the oral and nasal cavity. It prevents the virus from entering the cell of the human body, it prevents its multiplication. It has been observed that in COVID-19 patients, zinc associated with Plaqenil has given good results, the patients recovering faster. [9]

\section{Omega 3}

Used as a supplement in COVID-19, it prevents the production of inflammatory cytokines, reduces triglycerides and improves the liver function. 


\section{Melatonin}

The hormone synthesized in the body to regulate the sleep-wake cycle. It is produced by the pineal gland (epiphysis), an endocrine gland located at the brain level and by different tissues of various organs in the human body. The effect of its production is sleep, but it helps in COVID-19, having several effects: anti-inflammatory, antioxidant, viral antimodulator and even direct antiviral action. Because, with age decreases its synthesis in the body, it leads to the conclusion that the elderly people, affected by this disease, need a supplement of this hormone. [10,11]

\section{Herbal extracts with beneficial effect against CORONAVIRUS - COVID-19}

Recently, the benefits of some natural remedies, obtained by extraction from different plants, with therapeutic action against COVID-19 have been brought to a greater attention. Some examples will be described below.

\section{Boswellia serrata (incense)}

It is a resin of the serrata tree from India, used for therapeutic purposes. In COVID-19 helps at the reducing of the pain, fight cough, shortness of breath (dyspnea) and improves blood circulation.

\section{Valerian}

It is a plant used for the sedative effect, with small pink flowers, with a height of $1.5 \mathrm{~m}$, with a pleasant smell, but dry, it has an unpleasant smell; volatile oils give a calming effect. It also contains alkaloids, vitamin A, vitamin B1, vitamin B2, vitamin B6, vitamin C, vitamin $\mathrm{E}$, phosphorus, iron, magnesium, potassium, calcium and omega 3. Along with the calming effect, it fights insomnia, reduces anxiety, treats obsessive-compulsive disorder, compulsive, has an antibacterial effect. $[12,13]$

\section{Passiflora}

It is a plant used for calming and appeasement, inducing a balanced state of the psyche, reduces the states of fear and stress. It is indicated in COVID-19, during and after the disease, for chronic fatigue, depression, anxiety, panic attacks, arrhythmias, as well as for the peripheral circulatory system.

\section{Rhodiola}

It is a plant used as a natural nutritional supplement, which fights the stress and fatigue in the case of COVID-19, but for visible results it must be used for at least 12 weeks, with doses between 200 and $600 \mathrm{mg}$ / day. For depression, the dose is $340-680 \mathrm{mg} /$ day. Rhodiola Rosea, extract, is extremely effective, better than Ashawaganda, but after Panax Ginseng. [14, 15]

\section{Ashawaganda (Indian ginseng)}

Red or Indian ginseng, used by Ayurvedic medicine, is a tropical plant that comes from India, Asia and Africa, wild, with the name of winter cherry, in the form of a thick shrub, with yellow flowers and red fruits having the size of raisins. The therapeutic value is given, mainly by the root, which contains energy-stimulating substances: fatty acids, alkaloids, sugars and amino acids. The powder contains: protein, sugars, fiber and vegetable fats. It has anti-inflammatory, antioxidant, detoxifying properties, resulting in the sleep induction, body well-being, energy, longevity. It maintains mental health, being antidepressant and anti-stress. It is used for stress, nervous conditions, migraines, lack of concentration, brain degeneration, even for diseases such as Parkinson's and Alzheimer's. In COVID-19, it is used to relieve depression and anxiety in the post-COVID 19 phase, being very effective, as well as antidepressants and tranquilizers, resulting in a good sleep, reduces fatigue and well-being. To combat insomnia, use a teaspoon of powder in a glass of milk sweetened with honey or tea, 1-2 hours before bedtime. It is also used as an antibacterial in respiratory infections. It is forbidden to pregnant and lactating women. [16]

\section{Curcumin (turmeric)}

It is a plant widely used along with ginseng, garlic, ginger in various natural preparations, used as anti-inflammatory, anticoagulant, antidepressant, antibacterial, anticholesterol, antiglycemic. It has a potency result like antidepressant. 


\section{Echinacea}

It is one of the most known and used medicinal plants in the world, and of the 9 species there are only 3 species of medical interest: Echinacea purpureea, Echinacea pallida and Echinacea angustifolia. For Echinacea purpureea herba, the recommendations are for strengthening the immune system and supporting the body in colds and upper respiratory tract infections, flue infections.

\section{Wormwood}

Contains artemisinin, with hydroxychloroquine-like action, it is used as a drug in the treatment regimen of COVID-19, is a plant with antiviral, antibacterial, immunostimulatory and antipyretic effect.

\section{Oregano oil}

It is used internally in the nasopharyngeal cavity, preventing the entry of viruses into the body, being an effective natural antiviral and antibacterial. Nebulization or nasal washes, with sea water or plant extracts, helps to a better breathe in case of disease, leading to a significant increase in immunity of the affected body.

\section{Astha 15}

Natural product, a combination of 12 extracts and volatile oils, used for the health of mucous membranes (respiratory system), increasing the efficiency of the immune system, calming wheezing (dyspnea) and coughing. It comes in the form of capsules and syrup, administered in the morning and evening (two capsules / 2 teaspoons / day) with a sufficient quantity of water after a meal. It is used with good post-COVID-19 results, for a minimum period of 4 months.

Cough, one of the symptoms present at all patients with COVID-19, can be treated with: -dry cough- the combination of hemoderivative extraction of Honeysuckle with Hornbeam and Blackcurrant;

-productive cough- the combination of black poplar hemoderivative with Hornbeam and Blackcurrant. Hemoderivatives are administered as such, 25 drops in water, 2-3 times a day. Gemotherapy in rosehip branches contains a high quantity of ascorbic acid (vitamin C) and dehydroascorbic acid (vitamin P - to reduce the permeability and fragility of capillaries) and is one of the most used preparations in situations of exhaustion of the body, general impairment of immunity, relief of symptoms of respiratory tract infections, convalescence, post-flue and postinfectious asthenia. It can be administered together with extracts from Blackcurrant buds, birch buds, fir buds. [13-15]

\section{Conclusions}

In the context of the Covid-19 pandemic, of which dramatic consequences affects the population and economy of the entire planet, it is required a documentation on the treatments for COVID-19, including natural remedies, dietary supplements, plant substances and extracts associated with antiviral drugs, which have been shown to be effective in preventing and treating the SARS-CoV-2 infection. It is necessary for medical staff to be documented on these issues, so that, through interaction with the population, they can contribute to the joint effort to combat the pandemic. Medicinal plants are an important source of effective natural remedies for curing various diseases. The use of natural resources, especially plants with traditional value and dietary supplements (vitamins, trace elements) has been and is a major part of research aimed at associating them with antiviral drug treatment, in order to increase the immunity and prevent respiratory diseases. Since the outbreak of Covid-19 infection, numerous studies have argued that naturopathy should be used in combination with conventional medicine in the treatment of patients with Covid-19.

\section{References}

[1] Antimicrobial Agents: Antifungal \& Antiviral Drugs - Dr. (Ms) Naresh Khanna, Dept of Pharmacology University College of Medical Scientes, Shahdara, 2008. 
[2] Zhou M., Zhang X. Qu J., Coronavirus disease 2019 (COVID-19), a clinical update; Front med, aprilie 2020.

[3] https://depts.washington.edu/covid19trx/about/

[4] Mousavizadeh L., Ghasemi S., Genotype and phenotype of COVID-19: Their roles in pathogenesis, Journal of Microbiology, Immunology and Infection, aprilie 2021, Vol 54, Issue 2.

[5] Eyal, N., et al.: Human challenge studies to accelerate coronavirus vaccine licensure. The Journal of infectious diseases, 2020, 221(11).

[6] Cîrţînă D, Biochemistry, Ed. Academica Brâncuşi, Tg-Jiu, 2010.

[7] Cristea N., Clinical pharmacology, Ed. Medicală, Timișoara 2007.

[8] Tica A., Voicu V., Pharmacology, Editura Medicală Universitară Craiova, 2004.

[9] Derwand R., Scholz M., Does zinc supplementation increase the clinical efficacy of chloroquine / hydroxychloroquine to win the battle against COVID-19 today? Hypotheses Med. 2020; 142:109815.doi:10.1016/j.mehy.

[10] Fulga I. et al., Pharmacology, ediția a II-a, Editura Universul, București, 2017.

[11] Dobrescu D., Memomed, Ediția 27, Editura Universitară București, 2021.

[12] Ardelean A., Mohan Ghe. Medicinal flora of Romania, Editura All, București, 2008.

[13] Chirigiu, L. Bubulica, M.V. Averi, L.M.E. Investigations of three phytopharmaceutical products from Caprifoliaceae family using GC-MS and LC-MS, Revista De Chimie 63 (8), 764-768, 2012.

[14] https://www.plantextrakt.ro/

[15] https://www.drugs.com/npp/rhodiola-rosea.html

[15] https://www.healthline.com/nutrition/12-proven-ashwagandha-benefits\#1 